\title{
3D Scene Modeling and Rendering Algorithm Based on Road Extraction of Simple Attribute Remote Sensing Image
}

\author{
Zhiguang Wang ${ }^{*}$, Haiying Zhang, Qiang Lu and Yuanfeng Lian \\ Department of Computer Science and Technology, China University of Petroleum, Beijing, China \\ Beijing Key Laboratory of Petroleum Data Mining, China University of Petroleum, Beijing, China \\ ${ }^{*}$ Corresponding author
}

\begin{abstract}
In order to solve the problem of modeling and rendering of large data scene, this paper presents a fast method for modeling and rendering multi-level, multi-resolution road based on the road information extraction of simple attribute remote sensing image. Firstly, Fuzzy C-means Clustering (FCM), Bwareaopen, Mathematical Morphology and Markov Random Field (MRF) road extraction methods are used to extract the road skeleton information and road center control points of satellite remote sensing images. Then, the cubic B-spline curve function is used to fit the control information of the road center into the central line of the road. Finally, according to the terrain resolution levels, we will rebuilt the road model, which is rendered at multiple levels through the terrain resolution level by modifying the terrain data to achieve the effect of long-distance fuzzy and close-distance clearness. Experiments show that this method can realize the modeling and rendering of large data scenes effectively and vividly.
\end{abstract}

Keywords-remote sensing image; road extraction; multiresolution terrain; modeling rendering

\section{INTRODUCTION}

In recent years, road extraction of remote sensing images, reconstruction and rendering of three-dimensional virtual scenes are the hot spots. However, for the different types of input images, the research methods proposed in recent years lack stability and robustness in terms of extraction accuracy. For example, the Hough transform [1] method is based on the straight-line segmentation method to extract the roads, that is Hough method has high accuracy in the extraction of straightline roads; P-value segmentation [2] method need to set a threshold in advance and then extract the road, there will be more noises in the extracted image if this threshold was setting inappropriately. In order to improve the accuracy of road extraction for various shapes in remote sensing images, a hybrid road extraction method is proposed in this paper. Firstly, Fuzzy C-means Clustering (FCM) [3] is used to divide the image into multiple clusters of clustering pixels. Then the clustered image was processed by Bwareaopen [3] and Mathematical Morphology [4] to remove the salt-and-pepper noise and the large area noises in the image. Finally, we use Markov Random Field (MRF) [3] to extract the road in the road center. Thus, we can use the precise control points to fitting the whole road.
In addition, in the reconstruction and rendering of $3 \mathrm{D}$ virtual scene, the main research methods at home and abroad in recent years are "split-merge" and single-resolution terrain modeling and rendering. The so-called "split-merge" is independent rendering and then merging the two. For example, the constrained Delaunay triangulation method is used to integrate the terrain and linear vector elements to achieve the integrated display effect based on the DEM regular grid terrain in [5]. Reference [6] calculated the road control points by the bounding box algorithm, fitted the road structure with Bezier curve, and finally constructed the urban road model through the texture two-dimensional mapping. Reference [7] retrieved three-dimensional models according to images, semantic-based and sketch-based retrieval, and finally realized the rapid construction of 3D scene based on coordinate transformation. To a certain extent, these methods can achieve the road control point extraction and 3D scene reconstruction. However, when the amount of terrain data is particularly large (much larger than the memory capacity), terrain retrieval speed is very slow, computation is complicated, and it cannot achieve real-time performance, especially the multi-resolution terrain is basically not considered. Therefore, aiming at the large data volume and multi-resolution terrain scene, this paper proposes a fast and multi-resolution road modeling and rendering method based on regular grid topographic data, and realizes multi-level, multiresolution fast rendering in GPU framework.

\section{RoAd ExtRaction of Simple Attributes Remote SENSING IMAGE}

Simple Attributes Remote sensing images refer to the attributes contained in remote sensing images are relatively simple, such as roads and trees in this paper. Aiming at the few attributes characteristics of the remote sensing image data, the paper extracts the road information skeleton and the road center control points by combining FCM, Bwareaopen, Mathematical Morphology and MRF algorithm.

\section{A. Divides Road Pixels by FCM Clustering Method}

According to the characteristics of the image data of the selected simple attributes, the optimal number of clusters is 3 in this paper, that is, the road pixel, the tree outline pixel and the tree interior pixel. In our experiment, the membership factor of the optional parameters of FCM is 2, the maximum number of iterations is 200 and the termination criterion is $1 \mathrm{e}-5$. The simple attribute remote sensing image format selected is .jpg. 
The FCM clustering algorithm based on the above parameters is as follows:

Step1: Set the number of clusters c $(2 \leq \mathrm{c} \leq \mathrm{n})$, the membership factor $\mu(1<\mu)$, initial membership matrix $U_{r}(r=0)$, and the termination criterion $\varepsilon$.

Step2: Calculate the fuzzy cluster centers $\left\{\mathrm{C}_{\mathrm{i}} \mid \mathrm{i}=\right.$ $1,2, \cdots, c\}$ by using $U_{r}$.

Step3: Calculate the new partition matrix $\left\{\mathrm{C}_{\mathrm{i}} \mid \mathrm{i}=\right.$ $1,2, \cdots, c\}$ by using $\mathrm{U}_{\mathrm{r}+1}$.

Step4: Stop if $\left\|U^{r+1}-U^{r}\right\|=\max _{i, j}\left|u_{i, j}^{r+1}-u_{i, j}^{r}\right|<\varepsilon$, set $r=r+1$ and go to Step2.

As above, Step1 sets the constraint parameters (cluster number c, membership factor $\mu$ and initial membership matrix $U_{0}$ ) and ending condition (termination criterion $\varepsilon$ ) of FCM algorithm. Step2 calculates the corresponding cluster center $C_{i}$ by using the set initial membership matrix $U_{r}$; Step3 calculates the corresponding membership degree matrix $U_{r+1}$ by using the calculated cluster centers $\mathrm{C}_{\mathrm{i}}$. Step4 is the judgment of the loop condition or the end condition, if Step2 and Step3 calculate the convergence, the iteration is over, otherwise continues the iteration.

FCM clustering results are shown in Figure 1, map (a) represents the original image; map (b) represents the class diagram of Cluster 1 (outline pixel map of tree), map (c) represents the class diagram of Cluster 2 (internal pixel map of tree ), and map (d) represents the class diagram of Cluster 3 (road pixel map). As can be seen from map (d), most of the road parts are well preserved as well as separated from others.

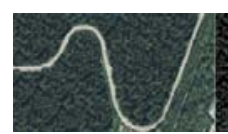

(a)

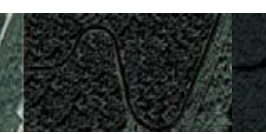

(b)

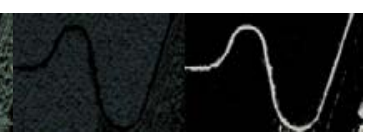

(c)

(d)
FIGURE I. FCM CLUSTERING RESUKTS: (A)ORIGINAL, (B) CLUSTER ONE DIAGRAM, (C) CLUSTER TWO DIAGRAM, (D) CLUSTER THREE DIAGRAM

\section{B. MRF Method to Extract Road Control Points}

As it can be seen from Figure 1 (d), there are still many salt-and-pepper and large-area noises in the image sorted by the FCM method. In order to reduce the noises, Bwareaopen and Mathematical Morphology algorithms are used, as shown in Figure 2 (c). It can be seen that after de-noising Figure 2 (c) is clearer than the image 1 (d). However, the road image processed by denoising always exists as a road image with a certain width, if the image is directly used for extracting road control points, it will have relatively large extraction error. Therefore, we use road center skeleton network function of MRF to accurately extract the centerline of the road skeleton. Firstly, the image is processed by the average sampling process algorithm, the sampled image pixels are $47 p x^{*} 26 p x$, then we traverse the sampled image pixels taking the $\mathrm{x}$-axis coordinate pixels as traversal object, taking its corresponding y coordinate pixel every 3 pixels, and finally we get the coordinates of the road center control points. Figure 2 (a) shows the original image, Figure 2.2 (b) shows the road pixel map divided by FCM method, and Figure 2 (c) shows the road pixel extracted from the noise removal method on the basis of Figure 2 (b).
Figure $2(\mathrm{~d})$ shows the road network image extracted by MRF. The extracted road control points are shown in Table 1 . The 16 points in the table are the control points obtained by sampling and traversing the sampled image according to the mean value of the graph Figure 2 (d). That is, the obtained control points can be used to fit entire road in the simple attribute remote sensing image.

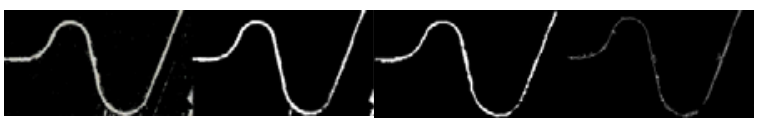

(a)

(b)

(c)

(d)

FIGURE II. ROAD NETWORK EXTRATION RESUKTS: (A)FCM CLUSTERING CLASSIFICATION DIAGRAM, (B) FIRST DENOISING RESULT DIAGRAM, (C) SECOND DE-NOISING DIAGRAM, (D) ROAD NETWORK DIAGTAM

TABLE I. EXTRACTED ROAD PIXEL CONTROL POINTS

\begin{tabular}{|c|c|c|c|c|c|}
\hline Number & X value & Y value & Number & X value & Y value \\
\hline 1 & 1 & 14 & 9 & 25 & 4 \\
\hline 2 & 4 & 14 & 10 & 28 & 1 \\
\hline 3 & 7 & 13.5 & 11 & 31 & 0 \\
\hline 4 & 10 & 16 & 12 & 34 & 1 \\
\hline 5 & 13 & 20 & 13 & 37 & 6 \\
\hline 6 & 16 & 23 & 14 & 40 & 14 \\
\hline 7 & 19 & 22.5 & 15 & 43 & 22 \\
\hline 8 & 22 & 18.5 & 16 & 46 & 26 \\
\hline
\end{tabular}

\section{CREATE THE ROAD MODEL}

Based on the existing data set of the control points of the road centerline, the cubic B-spline curve algorithm is used to fit the centerline of the road, and then the road model is established and generated by setting the parameters (the road width $\mathrm{W}_{\mathrm{f}}$ and the road height height). The establishment of the road model is achieved with the resolution of the terrain. In this paper, there are a total of 8 terrain layers and the road and terrain multi-resolution matching algorithm is as follows:

Step1: Based on the existing control points of the road center, cubic B-spline curve is used to fit the curve of the road center.

Step2: Calculate the distance dist from sample center to the center curve.

Step3: Set the half width $W_{f}$ and height height of the road.

Step4: If the terrain layer is in 1 to 5 layers, does not change the terrain elevation data, to Step10; if it is in the 6 to 8 layers, then to Step5.

Step5: The sampling points around the terrain are on the road model, to Step6; otherwise, to Step10.

Step6: If dist is less than $0.8 W_{f}$, to Step7; dist is less than $W_{f}$ and greater than $0.8 W_{f}$, to Step8; and dist is greater than $W_{f}$, go to Step9.

Step7: Modifying the road elevation, to Step10.

Step8: Modifying the road shoulder elevation, to Step10.

Step9: Modifying the road slope elevation, to Step10.

Step10: End. 
In the above algorithm, the terrain data does not need to be modified when the terrain is at a low resolution (for example, from 1 to 5 layers), that is, no road model needs to be established. When the terrain is at a high resolution (from 6 to 8 layers), the parameters ( $W_{f}$, height, dist, etc.) will be used to achieve the establishment of the road model, and do the following elaborating.

\section{A. Cubic B-spline Algorithm Fits Centerline}

The road center control points are extracted from the single attributes remote sensing images through the above FCM clustering, de-noising processing and MRF regularization methods. Based on the existing set of control points in road center, cubic B-spline curve is used to fit the center line of the road. B-spline curve is defined as follows:

$$
\begin{aligned}
P(u) & =\sum_{i=0}^{n} P_{i} B_{i, k}(u), u \in\left[u_{k-1}, u_{n+1}\right] \\
\mathrm{B}_{\mathrm{i}, \mathrm{k}}(\mathrm{u}) & =\frac{1}{\mathrm{k} !} \cdot \sum_{\mathrm{r}=0}^{\mathrm{k}-\mathrm{i}}(-1)^{\mathrm{r}} \mathrm{C}_{\mathrm{k}+1}^{\mathrm{r}}(\mathrm{t}+\mathrm{k}-\mathrm{i}-\mathrm{r})^{\mathrm{k}} \\
\mathrm{i} & =0,1, \ldots, \mathrm{k} \\
P_{i, k}(u) & =r * \partial * M * T
\end{aligned}
$$

In the above (3), the value of $\mathrm{i}$ is $0, \mathrm{k}$ is $3, \mathrm{r}$ is $1 / 6, \partial$ is $\left[1, u, u^{2}, u^{3}\right]$, the $M$ matrix is $\left[\begin{array}{cccc}1 & 4 & 1 & 0 \\ -3 & 0 & 3 & 0 \\ 3 & -6 & 3 & 0 \\ -1 & 3 & -3 & 1\end{array}\right], T$ is $\left[\mathrm{P}_{0}, \mathrm{P}_{1}, \mathrm{P}_{2}, \mathrm{P}_{3}\right]^{\mathrm{T}}, \mathrm{u} \in[0,1]$.The implementation of this algorithm in Matlab2012a is shown in Figure 3.

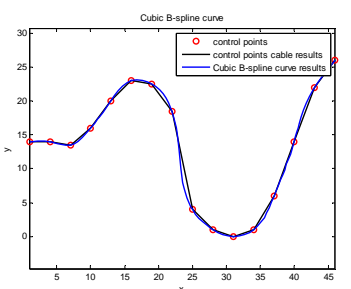

FIGURE III. CUBIC B-SPLINE CURVE FITTING DISPLAYS

\section{B. Road Model Design}

As mentioned above, the control points of the road center are converted into the central curve of the road by cubic Bspline curve fitting. In order to conform the conventional road model, it is necessary to set the half width $W_{f}$ and the height height of the road first, and then calculate the distance dist from the center curve of the road in the coordinate of the terrain data. If dist is on the designed road model, the terrain coordinate value is used to reconstruct the road model as shown in formula (4) below.

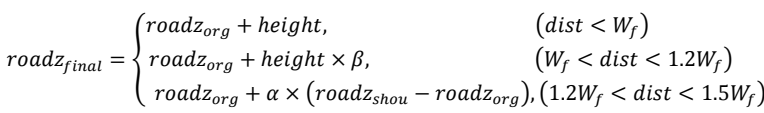

Which roadz $z_{\text {final }}$ is the final elevation of the road value, roadz $_{\text {org }}$ is the road elevation value of the original, roadz $z_{\text {shou }}$ is the road slope elevation value, height is the road height value, $\beta$ is the elevation ratio parameters of roadz $\mathrm{shou}_{\text {and }} \mathrm{roadz}_{\text {org }}$, and $\alpha$ is slope parameter. The design of the road cross-section is shown in Figure 4. The roads in the figure are divided into the road surface (lane and central divider), the shoulder and the slope, and the 3D cross-section of the road is shown in Figure 5.

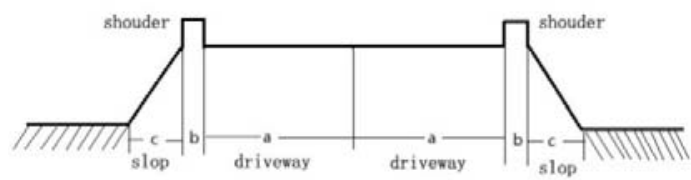

FIGURE IV. ROAD CROSS-SECTIONAL SCHEMATIC

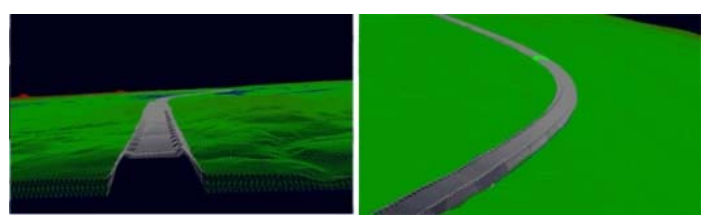

FIGURE V. ROAD CROSS-SECTION DESIGN RENDERINGS

\section{MULTI-LEVEL ROAD RENDERING}

Road multi-level rendering is designing different types of roads rendering based on the multi-resolution terrain. There are three ways to render a road: linear rendering, color rendering, and texture mapping rendering. Different rendering results of roads are invoked to achieve different display effect according to the distance of sight. The specific algorithm is as follows.

Step1: 3D terrain scene shows the result.

Step2: Terrain layer is in 1 to 3 layers, to Step3; in 4 to 5 , to Step4; in 6 to 8 layers, to Step5.

Step3: Road linear rendering, to Step6.

Step4: Road color rendering, to Step6.

Step5: Road texture rendering, to Step6.

Step6: End.

In the above algorithm, the linear road rendering algorithm sets the width of the road on the premise of knowing the central curve of the road, and then sets the property of the width of the road by using the sampling point distance. Finally, we render the road by the fragment shader; color road like linear road, there is no need to modify elevation data for terrain, but rendering color roads is relatively complicated. First, we set the width of the road according to the central curve and then set the properties of the road according to the sampling point distance, including the white solid line at the road edge line and the color of the road surface. Finally, in the fragment shader, different road colors are rendered according to the different attributes of the road to achieve the simulation effect of the real road. The texture road rendering method is based on the road model for texture rendering. First we bind the texture in the disk to the texture object and then transform the texture to the fragment shader. Finally, we find the coordinates of the texture rendering and render the texture to the surface of the road model. The specific result is shown in Figure 6. The figure (a) shows the road linear rendering results, Figure (b) shows the color road rendering results, and Figure (c) shows the texture road rendering. 


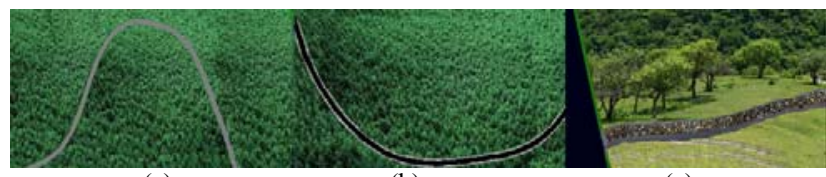

(a)

(b)

(c)

FIGURE VI. ROAD RENDERING RESULTS: (A) LINEAR ROAD RENDERING, (B) COLOR ROAD RENDERING, (C) TEXTURE ROAD RENDERING

\section{EXPERIMENTAL COMPARATIVE ANALYSIS}

The experimental platform used in this paper is as follows: Intel Core i5-2400 CPU, 3.1GHz CPU, 4GB RAM, Windows 10 x64 operating system, OSG2.8.2 development language, Matlab2012a and Visual Studio 2010. The experimental data that is the large scale terrain elevation texture data provided by the Bureau of Geophysical Prospecting. The comparison between the proposed method in this paper and the "splitmerge" scene integration approach in algorithm efficiency is shown in Table 2 and Figure 7. As it can be seen from the graph, the multi-resolution road scene integration frame rate is very fast at 1 and 3 layer, reaching $60 \mathrm{fps} / \mathrm{s}$, reducing the frame rates at layer 5 and 7 to $53 \mathrm{fps} / \mathrm{s}$ and $47 \mathrm{fps} / \mathrm{s}$ respectively, But compared to the "split - merge" method, our method is still higher which reaches the efficiency of nearly $18 \mathrm{fps} / \mathrm{s}$. It can be seen that the method in this paper is very efficient in sceneintegrated modeling and rendering.

TABLE II. SCENE INTEGRATION EFFICIENCY COMPARISON RESULTS

\begin{tabular}{|c|c|c|}
\hline $\begin{array}{c}\text { Terrain } \\
\text { layers }\end{array}$ & $\begin{array}{c}\text { Split-Merge frame } \\
\text { rate(fps) }\end{array}$ & $\begin{array}{c}\text { Multi-resolution road } \\
\text { frame rate(fps) }\end{array}$ \\
\hline L1 & $>=47$ & $>=60$ \\
\hline L3 & $>=40$ & $>=60$ \\
\hline L5 & $>=35$ & $>=53$ \\
\hline L7 & $>=30.02$ & $>=47$ \\
\hline
\end{tabular}

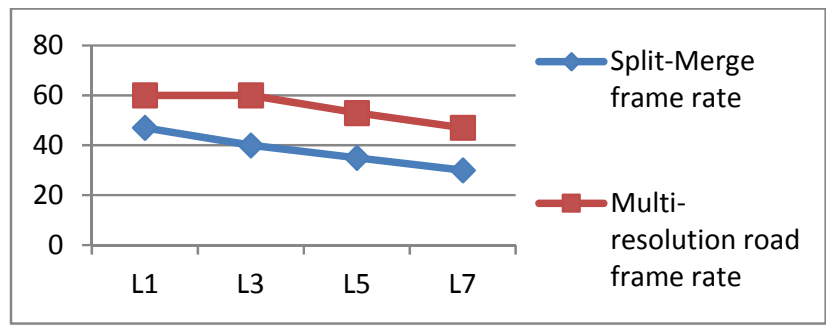

FIGURE VII. CONCLUSION SCENE INTEGRATION EFFICIENCY COMPARISON LINE CHART

\section{CONCLUSION}

This paper presents a fast and multi-level, multi-resolution road modeling method based on the recognition and extraction of simple attribute remote sensing images and the large terrain data. At the same time, it implements multi-level, multiresolution fast rendering. Experiments show that the algorithm in this paper is very efficient. Although the algorithm in this paper has achieved good results both in realism and real-time interaction, this article does not consider the modeling of multiple complex roads, so in the future research, we will extract the multiple roads and model them.

\section{ACKNOWLEDGMENT}

The research is supported by the National "973" project of China (No. 2013CB228602).

\section{REFERENCES}

[1] Xiaodan Liu, Yan Liu. Urban Road Extraction Based on Hough Transform and Path Morphology[J]. Computer Engineering and Applications, 2012,38(6):265-268.

[2] Jiawei Xu, "Improvement of SVM-based Road Extraction from Remote Sensing Image by using FCM and MRF” [D]. Hallym University, 2010.

[3] DM Zhu, X Wen, CL Ling. Road Extraction Based on the Algorithms of MRF and Hybrid Model of SVM and FCM[J]. International Symposium on Image \& Data Fusion, 2011, 1-4.

[4] Praveena K S, V.J Nyamathi,. Extraction of Road Network from Aerial and Satellite Images using Mathematical Morphology[J]. International Journal for Scientific Research \& Development, 2015:1073-1076.

[5] Mianmian Cheng, Shaomei Li, Xinmin Zhu, Jianqiao Chen. Three Dimensional Visualization Method Based on Regular Grid DEM Linear Vector [J]. Journal of Surveying and Mapping Engineering, 2015, 24(2):38-41.

[6] Xianlong Wang, Youquan Liu, Haoyu Li, Fei Dong. Automatic Construction of Roads in Large 3D Traffic Scene [J]. Journal of Computer Simulation, 2016, 33(10):124-128.

[7] Xiaohui Tan, Yachun Fen, XuesongWang, Mingquan Zhou. Modeldriven rapid $3 \mathrm{D}$ scene construction [J]. Journal of System Simulation, 2013, 25(10):2397-2402.

[8] Chenhao Wang, Xiaoan Tang, Jixiang Sun, Hong Chen. Road Generation and Mapping Based on Displacement Mapping [J]. Journal of System Simulation, 2011, 23(9):1905-1908.

[9] Aixia Zhou, Li Yu, Jiang Fen, Xueyan Zhang. Research on High Resolution Remote Sensing Image Road Extraction Based on Object Oriented Method [J]. Journal of Surveying and Mapping and Geospatial Information Science, 2017, 40(2):1-4.

[10] Bo Li, Rong Dong, Qimei Chen. Automatic Calibration Method for PTZ Camera [J]. Journal of Beijing University of Posts \& Telecommunications, 2009, 32(s1):24-29. 